MODELING, IDENTIFICATION AND CONTROL, 1991, VOL. 12, NO. 3, 129-138

doi:10.4173/mic.1991.3.3

\title{
Sliding control of MIMO nonlinear systems
}

\author{
THOR I. FOSSEN $\dagger$ and BJARNE A. FOSS $\dagger$
}

Keywords: Feedback linearization, sliding control, Lyapunov stability theory, polymerization reactor.

\begin{abstract}
Sliding control of MIMO (multivariable input-multivariable output) nonlinear minimum phase systems is discussed. Stability conditions related to model errors are emphasized. Global asymptotic stability is guaranteed by applying Barbalat's Lyapunov-like lemma. The control law is applied to a simulator of a polymerization reactor.
\end{abstract}

\section{Introduction}

The major problem with feedback linearization techniques is robustness due to imprecise cancellations of the model nonlinearities. In the case of parametric uncertainties, global asymptotically stable controllers may be found by using the Lyapunov stability theory. Well known techniques, arising from this approach are adaptive feedback linearization and sliding control which both have been successfully applied in robotic control (Slotine (1983)). Sliding control has also been applied in the control of underwater vehicles which are highly nonlinear and time-varying in their parameters (Yoerger and Slotine (1985)). This paper shows how sliding control can be applied to MIMO nonlinear minimum phase systems in the form $\dot{\boldsymbol{x}}=\boldsymbol{f}(\boldsymbol{x})+\boldsymbol{G}(\boldsymbol{x}) \boldsymbol{u}$ with $\boldsymbol{y}=\boldsymbol{h}(\boldsymbol{x})$. These results are also extended to the more general model class $\dot{\boldsymbol{x}}=\boldsymbol{f}(\boldsymbol{x}, \boldsymbol{u})$ with $\boldsymbol{y}=\boldsymbol{h}(\boldsymbol{x})$. In some cases, the general model class is necessary because of the complex structure of a model. This is true in certain process control applications, for instance. An example from a polymerization plant will be used in this investigation.

The paper is outlined as follows: Section 2 discusses feedback linearization and sliding control of MIMO affine systems. Sliding control of the more general model class is examined in Section 3, while results from the simulation studies are presented in Section 4. The paper ends with the conclusions.

\section{Affine systems}

Before we discuss the more general nonlinear model class $\dot{\boldsymbol{x}}=\boldsymbol{f}(\boldsymbol{x}, \boldsymbol{u})$ we will consider MIMO nonlinear systems which are linear in control or affine. These systems can be expressed as

$$
\begin{aligned}
& \dot{x}=f(x)+G(x) u \\
& y=h(x)
\end{aligned}
$$

with $\left(x \in \mathscr{R}^{n}, y \in \mathscr{R}^{m}, u \in \mathscr{R}^{p}\right)$ and $(f(x), G(x)$ and $h(x))$ smooth.

Received 11 November 1991.

$\dagger$ Division of Engineering Cybernetics, The Norwegian Institute of Technology, N-7034 Trondheim, Norway.

This paper was presented at the European Control Conference, Grenoble, France, 2-5 July 1991. Reprinted with permission from Editions Hermés. 
2.1. Review of input-output feedback linearization of affine systems

Differentiating the output $\mathbf{y}$ with respect to time yields

$$
\dot{y}=H_{x}(x) f(x)+H_{x}(x) G(x) u
$$

where $\boldsymbol{H}_{\boldsymbol{x}}(\boldsymbol{x})=\partial \boldsymbol{h}(\boldsymbol{x}) / \partial \boldsymbol{x}$ is the Jacobian. This control problem may be reduced to that of controlling the linear system

$$
\dot{\boldsymbol{y}}=\boldsymbol{v}
$$

where the choice: $v=\dot{y}_{d}-K_{P}\left(y-y_{d}\right)$ yields the error equation

$$
\dot{e}+K_{p} e=0
$$

Here $\boldsymbol{K}_{\boldsymbol{P}}$ is a positive definite design matrix of appropriate dimension, $\boldsymbol{y}_{d}$ is the desired trajectory and $\boldsymbol{e}=\boldsymbol{y}-\boldsymbol{y}_{\boldsymbol{d}}$. If the system Eqn. 1 is square, for example $m=p$, and $\boldsymbol{H}_{x}(x) G(x)$ is nonsingular for all $x \in \mathscr{R}^{n}$ the actual control input $u$ could be calculated as

$$
u=\left(H_{x}(x) G(x)\right)^{-1}\left[v-H_{x}(x) f(x)\right]
$$

It is convenient to let $r_{j}$ denote the systems relative degree i.e. the smallest number of differentiations $r_{j}$ the output $y_{j}$ has to be differentiated for one of the control inputs to appear. The total relative degree is defined as $r=r_{1}+\ldots+r_{m}$. Let us consider the case of $r<n$. Defining $G=\left[g_{1}, \ldots, g_{p}\right]$ and $L_{f} h_{j}$ and $L_{g_{j}} h_{j}$ as the Lie derivatives of $h_{j}$ with respect to $\boldsymbol{f}$ and $\boldsymbol{g}_{\boldsymbol{j}}$, respectively, implies that Eqn. 1 can be expressed as Sastry and Isidori (1989)

$$
y_{j}^{\left(r_{j}\right)}=L_{f}^{r_{j}} h_{j}+\sum_{i=1}^{m} L g_{i}\left(L_{f}^{r_{j}-1} h_{j}\right) u_{i}
$$

The smallest integer $r_{j}$ is found by requiring that at least one of the Lie derivatives $\mathbf{L} \boldsymbol{g}_{i}\left(L_{\boldsymbol{f}}^{r_{j}^{-1}}\right) \neq 0 \forall \boldsymbol{x}$. Notice that if the control input does not appear after at least $r_{j}$ differentiations the system will not be controllable. Let the $m \times m$ decoupling matrix $G^{*}(x)$ be defined as, Sastry and Isidori (1989),

$$
G^{*}(x)=\left[\begin{array}{ccc}
L_{g_{1}}\left(L_{f}^{r_{1}-1} h_{1}\right) & \ldots & L_{g_{m}}\left(L_{f}^{r_{1}-1} h_{1}\right) \\
\vdots & & \vdots \\
L g_{1}\left(L_{f}^{r_{m}-1} h_{m}\right) & \ldots & \operatorname{Lg}_{m}\left(L_{f}^{r_{m}-1} h_{m}\right)
\end{array}\right]
$$

and

$$
f^{*}(x)=\left(L_{f}^{r_{1}} h_{1}, \ldots, L_{f}^{r_{m}} h_{m}\right)^{\mathrm{T}}
$$

Hence, Eqn. 3 can be written in a compact form as

$$
\left[\begin{array}{c}
y_{1}^{\left(r_{1}\right)} \\
\vdots \\
y_{m}^{\left(r_{m}\right)}
\end{array}\right]=\boldsymbol{f}^{*}(\boldsymbol{x})+\boldsymbol{G}^{*}(\boldsymbol{x})\left[\begin{array}{c}
u_{1} \\
\vdots \\
u_{m}
\end{array}\right]
$$

The equivalent linear system to be controlled is

$$
y^{\left(\boldsymbol{r}_{j}\right)}=v_{j} ; \quad j=1 \ldots m
$$

If $G^{*}(x)$ is non-singular the nonlinear feedback control law

$$
u=\left(G^{*}(x)\right)^{-1}\left[v-f^{*}(x)\right]
$$


yields the decoupled system Eqn. 5 directly. A system with relative degree $\left(r_{1}, \ldots, r_{m}\right)$ can be transformed into a so-called normal form by applying a diffeomorphism $(\zeta, z)=T(x)$ defined as

$$
\begin{array}{cccc}
\zeta_{1}^{1}=h_{1} & \zeta_{2}^{1}=L_{\boldsymbol{f}} h_{1} & \ldots & \zeta_{\boldsymbol{r}_{1}}^{1}=L_{\boldsymbol{f}}^{r_{1}-1} h_{1} \\
\vdots & & & \vdots \\
\zeta_{1}^{m}=h_{m} & \zeta_{2}^{m}=L_{\boldsymbol{f}} h_{m} & \ldots & \zeta_{\boldsymbol{r}_{m}}^{m}=L_{\boldsymbol{f}}^{r_{m}-1} h_{m}
\end{array}
$$

This implies that Eqn. 4 can be expressed as

$$
\begin{aligned}
& \dot{\zeta}_{1}^{j}=\zeta_{2}^{j} \\
& \vdots \\
& \dot{\zeta}_{r_{j}}^{j}=f_{j}^{*}(\zeta, z)+\sum_{i=1}^{m} G_{j i}^{*}(\zeta, z) u_{i} \\
& y_{j}=\zeta_{1}^{j}
\end{aligned}
$$

where $j=1,2, \ldots, m$ and

$$
\dot{z}=\psi(\zeta, z)+\Psi(\zeta, z) u
$$

The state vector $z$ denotes the internal dynamics and

$$
\begin{gathered}
\psi_{k}(\zeta, z)=L_{f} \zeta_{k}(x) \\
\Psi_{k i}(\zeta, z)=L g_{i} \zeta_{k}(x)
\end{gathered}
$$

where $(k=1, \ldots, n-r)$ and $(i=1, \ldots, m)$. The zero-dynamics of the nonlinear system is defined as the dynamics of the system when the outputs are constrained to be identically zero i.e. $\zeta=0$. This is obtained by choosing the control inputs as

$$
u(0, z)=-\left[G^{*}\left(T^{-1}(0, z)\right)\right]^{-1} f^{*}\left(T^{-1}(0, z)\right)
$$

Eliminating $\boldsymbol{u}$ from Eqn. 7 yields the zero dynamics

$$
\dot{z}=\psi(0, z)-\Psi(0, z)\left[G^{*}\left(T^{-1}(0, z)\right)\right]^{-1} f^{*}\left(T^{-1}(0, z)\right)
$$

Notice that the zero dynamics are made unobservable by state feedback. The nonlinear system Eqn. 1 is said to be non-minimum phase if the zero dynamics are unstable and asymptotically minimum phase if the zero dynamics are asymptotically stable (Byrnes and Isidori (1984)). For minimum phase systems, feedback linearization results in bounding tracking if the desired motion trajectory is bounded. The proof is given in Sastry and Isidori (1989).

Uncertainties in the functions $\boldsymbol{f}$ and $\boldsymbol{G}$ in the non-linear decoupling may result in imprecise cancellations of the nonlinear terms. In the next section we will demonstrate how sliding control can be used to compensate for model uncertainties.

\subsection{Sliding control of affine systems}

The sliding design methodology is described in detail by Slotine $(1983,1991)$ and Utkin (1977). In the following, it is convenient to define the operators:

$$
\begin{aligned}
|x| & =\left[\left|x_{1}\right|,\left|x_{2}\right|, \ldots,\left|x_{n}\right|\right]^{\mathrm{T}} \\
\operatorname{sgn}(x) & =\left[\operatorname{sgn}\left(x_{1}\right), \operatorname{sgn}\left(x_{2}\right), \ldots, \operatorname{sgn}\left(x_{n}\right)\right]^{\mathrm{T}} \\
x . \times y & =\left[x_{1} y_{1}, x_{2} y_{2}, \ldots, x_{n} y_{n}\right]^{\mathrm{T}}
\end{aligned}
$$


In sliding control the error equation, Eqn. 2, is replaced by

$$
\dot{s}+k . \times \operatorname{sgn}(s)=w(t)
$$

where $s$ is a measure of tracking and $w(t)$ represents the external signals. According to the above definitions, $\boldsymbol{k} \times \operatorname{sgn}(s)$ yields a vector of elements $\boldsymbol{k}_{i} \operatorname{sgn}\left(s_{i}\right)$. If all $k_{i}$ satisfy $k_{i}>\sup _{t}\left|w_{i}(t)\right|$, then $s$ goes to zero in finite time. Define a sliding surface (see Slotine and Li 1987)).

$$
s_{j}=\left(\frac{d}{d t}+\lambda_{j}\right)^{r_{j}-1} e_{j} \text { where } e_{j}=y_{j}-y_{j, d}
$$

where $\lambda_{j}$ is a positive scalar specifying the control bandwidth and $y_{j, d}$ is the desired trajectory. For systems of relative degree $r_{j}=1$, Eqn. 8 simply yields $s_{j}=e_{j}$. Define a virtual reference vector $\alpha_{d}$ such that

$$
\dot{s}_{j}=y_{j}^{\left(r_{j}\right)}-\alpha_{d, j} ; j=1, \ldots, m
$$

For the system, Eqn. 4, we propose a control law

$$
u=\left(\hat{G}^{*}(x)\right)^{-1}\left[\alpha_{d}-\hat{f}^{*}(x)-k \cdot \times \operatorname{sgn}(s)\right]
$$

where the hat denotes the estimates of the nonlinear functions. The bounds of the elements $k_{i}$ may be derived by applying Barbalat's lemma. Notice, that the existence of $\boldsymbol{u}$ is influenced by the choice of the controlled variables $y$, i.e. the existence of the inverse of $G^{*}(x)$. Assume that the parametric uncertainties in the nonlinear functions $f^{*}$ and $G^{*}$ satisfy the following bounds

$$
\begin{gathered}
\left|\hat{\boldsymbol{f}}_{j}^{*}(\boldsymbol{x})-\boldsymbol{f}_{j}^{*}(\boldsymbol{x})\right| \leqslant \delta_{i} \\
\boldsymbol{G}^{*}(\boldsymbol{x})=(\boldsymbol{I}+\Delta) \hat{G}^{*}(\boldsymbol{x}) ; \quad\left|\Delta_{i j}\right| \leqslant D_{i j}
\end{gathered}
$$

where $(i=1, \ldots, m),(j=1, \ldots, m)$ and $\bar{\sigma}(\Delta)<1$. Consider a Lyapunov-like function candidate

$$
V(s, t)=\frac{1}{2} s^{T} s
$$
yields

Differentiating $V(s, t)$ with respect to time and substituting Eqns. 4, 9, 10 and 11

$$
\dot{V}=s^{\mathrm{T}} \dot{s}=s^{\mathrm{T}}\left[\left(f^{*}-\hat{f}^{*}\right)+\Delta\left(\alpha_{d}-\hat{f}^{*}\right)-(I+\Delta) k . \times \operatorname{sgn}(s)\right]
$$

From this it is seen that if $k_{i} \geqslant k_{i}^{\prime} \forall i$ where $k^{\prime}$ satisfies

$$
(\boldsymbol{I}-\overline{\mathbf{D}}) \boldsymbol{k}^{\prime}=\boldsymbol{\delta}+\mathbf{D}\left|\boldsymbol{\alpha}_{d}-\hat{\boldsymbol{f}}^{*}(\boldsymbol{x})\right|+\boldsymbol{\eta}
$$

where $\mathbf{D}$ is an $m \times m$ matrix with elements $D_{i j}$ and $\mathbf{D}$ is

$$
\overline{\mathbf{D}}=\left[\begin{array}{cccc}
D_{11} & -D_{12} & \cdots & -D_{1 m} \\
-D_{21} & D_{22} & & -D_{2 m} \\
\vdots & & \vdots & \vdots \\
-D_{m 1} & -D_{m 2} & \cdots & D_{m m}
\end{array}\right]
$$

the sliding condition

$$
\dot{V}=-\eta^{\mathrm{T}}|s|=\sum_{i=1}^{m}-\eta_{i}\left|s_{i}\right| \leqslant 0 ; \quad \eta_{i}>0
$$

Hence, applying Barbalat's Lyapunov-like lemma ensures that $s \rightarrow 0$ and thus $e \rightarrow 0$. 
3. Systems in the form: $\dot{x}=f(x, u)$ with $y=h(x)$

Although Eqn. 1 is a fairly general model description, not all systems can be modeled using this structure. For instance consider the following model class

$$
\dot{x}=f(x)+G_{1}(x) u+G_{2}(u) x
$$

which is nonlinear in the control input $u$. This justifies the use of a more general model class

$$
\begin{aligned}
& \dot{\boldsymbol{x}}=\boldsymbol{f}(\boldsymbol{x}, \boldsymbol{u}) \\
& \boldsymbol{y}=\boldsymbol{h}(\boldsymbol{x})
\end{aligned}
$$

Such a system will also be discussed in Section 4 in conjunction with the simulation of a polymerization reactor.

\subsection{Input-output linearization}

For simplicity let us first consider a system where $m=p$. Differentiating $\dot{y}$ in Eqn. 16 with respect to time yields

$$
\begin{aligned}
& \dot{y}=H_{x} f \\
& \ddot{y}=\frac{\partial}{\partial x}\left(H_{x} f\right) f+\frac{\partial}{\partial u}\left(H_{x} f\right) \dot{u}
\end{aligned}
$$

The nonlinear control law (assuming that $\partial / \partial \boldsymbol{u}\left(\boldsymbol{H}_{x} \boldsymbol{f}\right)$ is non-singular)

$$
\dot{u}=\left[\frac{\partial}{\partial u}\left(H_{x} f\right)\right]^{-1}\left[v-\frac{\partial}{\partial x}\left(H_{x} f\right) f\right]
$$

yields the linear system

which suggests $v$ to be chosen as

$$
\ddot{\boldsymbol{y}}=\boldsymbol{v}
$$

$$
v=\ddot{y}_{d}-K_{D} \dot{e}-K_{P} e
$$

where $\boldsymbol{e}=\boldsymbol{y}-\boldsymbol{y}_{d}$ and $\boldsymbol{K}_{D}$ and $\boldsymbol{K}_{P}$ are design matrices of appropriate dimensions chosen such that the error equation $\ddot{\boldsymbol{e}}+\boldsymbol{K}_{D} \dot{\boldsymbol{e}}+\boldsymbol{K}_{\mathrm{P}} \boldsymbol{e}=0$ is stable. In the general case, let $\gamma_{j}$ be the smallest number of differentiations of the output $y_{j}$ for one of the derivatives $\dot{u}_{i}$ to appear, then

or equivalently

$$
y_{j}^{\left(\gamma_{j}\right)}=L_{f}^{\gamma_{j}} h_{j}+\frac{\partial}{\partial u}\left(L_{f}^{\gamma_{j}-1} h_{j}\right) \dot{u} ; j=1, \ldots, m
$$

$$
\left[\begin{array}{c}
y_{1}^{\left(\gamma_{1}\right)} \\
\vdots \\
y_{m}^{\left(\gamma_{m}\right)}
\end{array}\right]=\overline{\boldsymbol{f}}(\boldsymbol{x}, \boldsymbol{u})+\boldsymbol{G}(\boldsymbol{x}, \boldsymbol{u})\left[\begin{array}{c}
\dot{u}_{1} \\
\vdots \\
\dot{u}_{m}
\end{array}\right]
$$

where $\bar{f}_{j}(x, u)=L_{f}^{\gamma_{j}} h_{j}(x)$ and

$$
\overline{\boldsymbol{G}}(\boldsymbol{x}, \boldsymbol{u})=\left[\begin{array}{ccc}
\frac{\partial}{\partial u_{1}}\left(L_{\boldsymbol{f}}^{\gamma_{1}-1} h_{1}\right) & \ldots & \frac{\partial}{\partial u_{m}}\left(L_{f}^{\gamma_{1}-1} h_{1}\right) \\
\vdots & & \vdots \\
\frac{\partial}{\partial u_{1}}\left(L_{f}^{\gamma_{m}-1} h_{m}\right) & \ldots & \frac{\partial}{\partial u_{m}}\left(L_{f}^{\gamma_{m}-1} h_{m}\right)
\end{array}\right]
$$


The nonlinear control law (assuming that $\overline{\boldsymbol{G}}(\boldsymbol{x}, \boldsymbol{u})$ is non-singular)

$$
\dot{\boldsymbol{u}}=(\bar{G}(x, u))^{-1}[v-\bar{f}(x, u)]
$$

yields $y_{j}^{\left(\gamma_{j}\right)}=v_{j}$ for $j=1, \ldots, m$.

\subsection{Sliding control}

The corresponding sliding control law is found by defining a sliding surface

$$
s_{j}=\left(\frac{d}{d t}+\lambda_{j}\right)^{\gamma_{j-1}} e_{j} \text { where } \gamma_{j}>1
$$

Let $\Lambda=\operatorname{diag}\left\{\lambda_{j}\right\}$. Hence, $\dot{V}$ can be written as

$$
\dot{V}=s^{\mathrm{T}} \dot{s}=s^{\mathrm{T}}\left(\bar{f}(x, u)+\bar{G}(x, u) \dot{u}-\alpha_{d}\right)
$$

This suggests the control law

$$
\dot{u}=\hat{G}(x, u)\left[\alpha_{d}-\hat{f}(x, u)-k . \times \operatorname{sgn}(s)\right]
$$

where the parametric uncertainties in the nonlinear functions are assumed to satisfy the following bounds

$$
\begin{gathered}
\left|\hat{\boldsymbol{f}}_{j}(\boldsymbol{x}, \boldsymbol{u})-\overline{\boldsymbol{f}}_{j}(\boldsymbol{x}, \boldsymbol{u})\right| \leqslant \delta_{j} \\
\overline{\boldsymbol{G}}(\boldsymbol{x}, \boldsymbol{u})=(\boldsymbol{I}+\Delta) \hat{\boldsymbol{G}}(\boldsymbol{x}, \boldsymbol{u}) ; \quad\left|\Delta_{i j}\right| \leqslant D_{i j}
\end{gathered}
$$

where $(i=1, \ldots, m),(j=1, \ldots, m)$ and $\bar{\sigma}(\Delta)<1$. Hence, $\dot{\mathbf{v}}$ can be written as

$$
\dot{\mathbf{V}}=s^{\mathrm{T}}\left[-(I+\Delta) k \times \operatorname{sgn}(s)+(\bar{f}-\hat{f})+\Delta\left(\alpha_{d}-\hat{f}\right)\right]
$$

From the expression for $\dot{V}$ it is easily seen that $\boldsymbol{k}$ must satisfy $k_{i} \geqslant k_{i}^{\prime} \forall i$ where:

$$
(I-\bar{D}) k^{\prime}=\delta+D\left|\alpha_{d}-\hat{f}(x, u)\right|+\eta
$$

with the matrix $\bar{D}$ defined as in Eqn. 14 to achieve $\dot{V} \leqslant-\eta^{\mathrm{T}}|\boldsymbol{s}| \leqslant 0$ which according to Barbalat's lemma implies that $s \rightarrow 0$ and thus $e \rightarrow 0$. Notice that the requirement that both $\boldsymbol{y}$ and $\dot{y}$ must be measured to calculate the measure of tracking $s$ is quite restrictive, especially in cases with noise on $\boldsymbol{y}$. If $\dot{\boldsymbol{y}}$ is not available from measurements, some algorithm for approximating $\dot{y}$ must be used. This is easy to realize if $\boldsymbol{y}$ is sampled at high sampling rate.

To avoid undesirable control chattering a saturation function sat $\left(s_{i} / \phi_{i}\right)$ can be used instead of $\operatorname{sgn}\left(s_{i}\right)$ to smooth out the control input inside a boundary layer (Slotine (1983)) where $\phi_{i}$ may be interpreted as the boundary layer thickness.

\section{Simulation study}

\subsection{Simplified model of polymerization reactor}

A simple two-stage polymerization reactor is illustrated in Fig. 1.

In the simulation study only the first stage of the process was considered. A more detailed model is found in Singstad (1991). This was done by using $x=\left[m, m_{p_{1}}, T_{1}\right]^{\mathrm{T}}$ as the state vector, $\boldsymbol{u}=\left[w_{m_{1}}, w_{i_{1}}, w_{f_{1}}\right]^{\mathrm{T}}$ as the input vector and $\boldsymbol{y}=\left[T_{1}, p\right]^{\mathrm{T}}$ as the output vector. $m$ is the total mass of the reactor, $m_{p_{1}}$ is the mass of polymer in stage $1, T_{1}$ is the temperature in the first stage, $w_{m_{1}}$ is the monomer feed rate, $w_{i}$ is the initiator feed rate 


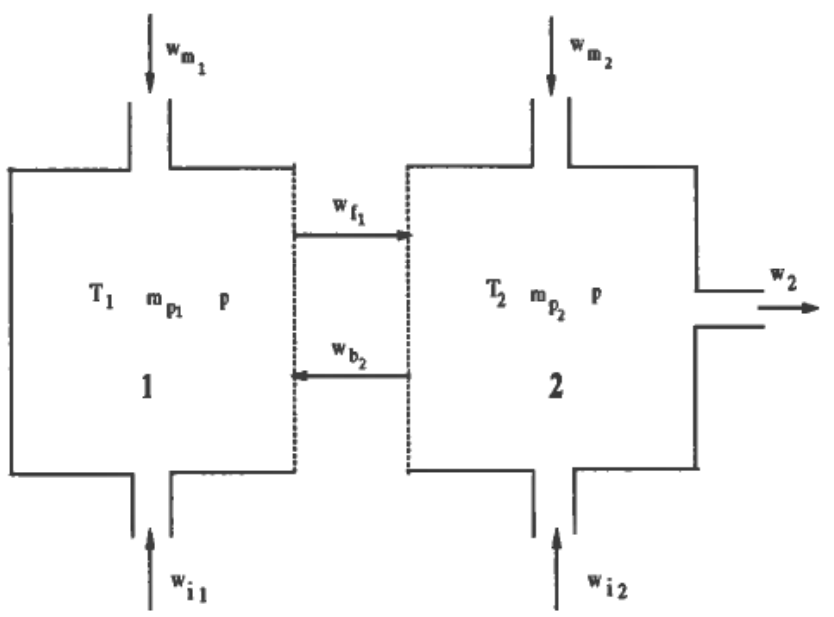

Figure 1. Two-stage polymerization reactor.

and $w_{f_{1}}$ is the output feed rate from stage 1 . For this system it is desirable to control the temperature $T_{1}$ and pressure $p$. For simplicity we assume that $u_{3}=u_{3_{s}}=$ const. Hence,

$$
\begin{aligned}
& \dot{x}=f(x, u) \\
& y=h(x)
\end{aligned}
$$

where

$$
\boldsymbol{f}(\boldsymbol{x}, \boldsymbol{u})=\left[\begin{array}{l}
u_{1}+u_{2} \\
a_{1}\left(u_{2}\right)^{1 / 2}\left(x_{1}-x_{2}\right)-\frac{x_{2}}{x_{1}} u_{3 s} \\
\frac{1}{x_{1}}\left(a_{1} a_{2}\left(u_{2}\right)^{1 / 2}\left(x_{1}-x_{2}\right)+u_{1}\left(a_{3}-x_{3}\right)\right)
\end{array}\right]
$$

and

$$
\boldsymbol{h}(\boldsymbol{x})=\left[x_{3}, a_{4} x_{1} x_{3}\right]^{\mathrm{T}}
$$

Differentiating $\boldsymbol{y}$ with respect to time $\left(\gamma_{j}=2\right)$ yields

$$
\begin{aligned}
& \dot{y}=H_{x} f \\
& \ddot{y}=\frac{\delta}{\delta x}\left(H_{x} f\right) f+\frac{\partial}{\partial u}\left(H_{x} f\right) \dot{u}
\end{aligned}
$$

where $\boldsymbol{H}_{\boldsymbol{x}}$ is the Jacobian

$$
\boldsymbol{H}_{x}=\left[\begin{array}{ccc}
0 & 0 & 1 \\
a_{4} x_{3} & 0 & a_{4} x_{1}
\end{array}\right]
$$

and

$$
\frac{\partial}{\partial \boldsymbol{u}}\left(\boldsymbol{H}_{x} \boldsymbol{f}\right)=\left[\begin{array}{cc}
\frac{a_{3}-x_{3}}{x_{1}} & \frac{a_{1} a_{2}\left(x_{1}-x_{2}\right)}{2\left(u_{2}\right)^{1 / 2} x_{1}} \\
a_{3} a_{4} & \frac{a_{1} a_{2} a_{4}\left(x_{1}-x_{2}\right)}{2\left(u_{2}\right)^{1 / 2}}+a_{4} x_{3}
\end{array}\right]
$$




$$
\begin{aligned}
& \frac{\partial}{\partial x}\left(H_{x} f\right) f=\left[\begin{array}{l}
-a_{1} a_{2} \frac{\left(u_{2}\right)^{1 / 2}}{x_{1}}\left(a_{1}\left(u_{2}\right)^{1 / 2}\left(x_{1}-x_{2}\right)-\frac{u_{3 s} x_{2}}{x_{1}}\right) \\
a_{1} a_{2} a_{4}\left(u_{2}\right)^{1 / 2}\left(u_{1}+u_{2}\right)-a_{1} a_{2} a_{4}\left(u_{2}\right)^{1 / 2}
\end{array}\right. \\
& -\frac{u_{1}}{x_{1}^{2}}\left(a_{1} a_{2}\left(u_{2}\right)^{1 / 2}\left(x_{1}-x_{2}\right)+u_{1}\left(a_{3}-x_{3}\right)\right) \\
& \times\left(a_{1}\left(u_{2}\right)^{1 / 2}\left(x_{1}-x_{2}\right)-\frac{u_{3 s} x_{2}}{x_{1}}\right) \\
& +\frac{u_{1}+u_{2}}{x^{2}}\left(-a_{3} u_{1}+a_{1} a_{2}\left(u_{2}\right)^{1 / 2} x_{2}+u_{1} x_{3}\right) \\
& +a_{4} \frac{u_{2}^{1}}{x_{1}}\left(a_{1} a_{2}\left(u_{2}\right)^{1 / 2}\left(x_{1}-x_{2}\right) u_{1}\left(a_{3}-x_{3}\right)\right)
\end{aligned}
$$

The constants were chosen as

$$
\begin{array}{ll}
a_{1}=0.04\left((\mathrm{kgs})^{-1}\right)^{1 / 2} & a_{3}=300 \mathrm{~K} \\
a_{2}=286 \mathrm{~K} & a_{4}=1950 \mathrm{~Pa} /(\mathrm{kg} \mathrm{K})
\end{array}
$$

In the simulation study the nominal model was simply perturbed as

$$
\begin{gathered}
\frac{\partial}{\partial x}\left(\widehat{H_{x} f}\right) \hat{f}=\left[\begin{array}{cc}
1 \cdot 5 & 0 \\
0 & 0.5
\end{array}\right] \frac{\partial}{\partial x}\left(H_{x} f\right) f \\
\frac{\partial}{\partial u}\left(\widehat{H_{x} f}\right)=(I+\Delta) \frac{\partial}{\partial u}\left(H_{x} f\right) ; \quad \Delta=\left[\begin{array}{rr}
-0.5 & 0.6 \\
-0.3 & -0.4
\end{array}\right]
\end{gathered}
$$

The control law was calculated from Eqn. 18 as

$$
\dot{u}=\left[\frac{\partial}{\partial u}\left(\widehat{H_{x} f}\right)\right]^{-1}\left[\ddot{y}_{d}+\lambda\left(\dot{y}_{d}-\dot{y}\right)-\frac{\partial}{\partial x}\left(\widehat{H_{x} f}\right) \hat{f}-k \cdot \times \operatorname{sgn}(s)\right]
$$

To avoid chattering the control law was smoothed out in a boundary layer with constant thickness i.e. $f=\left[\begin{array}{ll}0.4 & 0-4\end{array}\right]^{\mathrm{T}}$ by simply replacing the signum function with a sat $(\cdot)$ function defined as

$$
\operatorname{sat}\left(s_{i} / \phi_{i}\right)=\left\{\begin{array}{rll}
-1, & \text { if } & s_{i} \leqslant \phi_{i} \\
s_{i} / \phi_{i}, & \text { if } & -\phi_{i}<s_{i}<\phi_{i} \\
1, & \text { if } & s_{i} \geqslant \phi_{i}
\end{array}\right.
$$

The desired output vector was simply chosen as $\dot{y}_{d}+\alpha y_{d}=\alpha \boldsymbol{r}$ where $\alpha$ is a positive constant and $\boldsymbol{r}$ is the commanded input vector. The simulation was performed with two different controllers: (1) a nominal controller (switching gain: $k=0$ ) and (2) a robust sliding controller) switching gain: $\boldsymbol{k} \neq 0$ ). The simulation results for a sampling rate of $2 \mathrm{~Hz}$ are shown in Figs. 2-3. It is seen from the time responses that the sliding controller yields good performance while the tracking for the nominal controller is poor. The sliding controller compensates for model uncertainties, while the nominal case is strongly affected by imprecise cancellations of model nonlinearities. In the nominal case an undesirable chattering in one of the control efforts is also seen. Simulations showed that the difference between the nominal controller and the sliding controller varied according to changes in the operating conditions. In all cases however, we found 

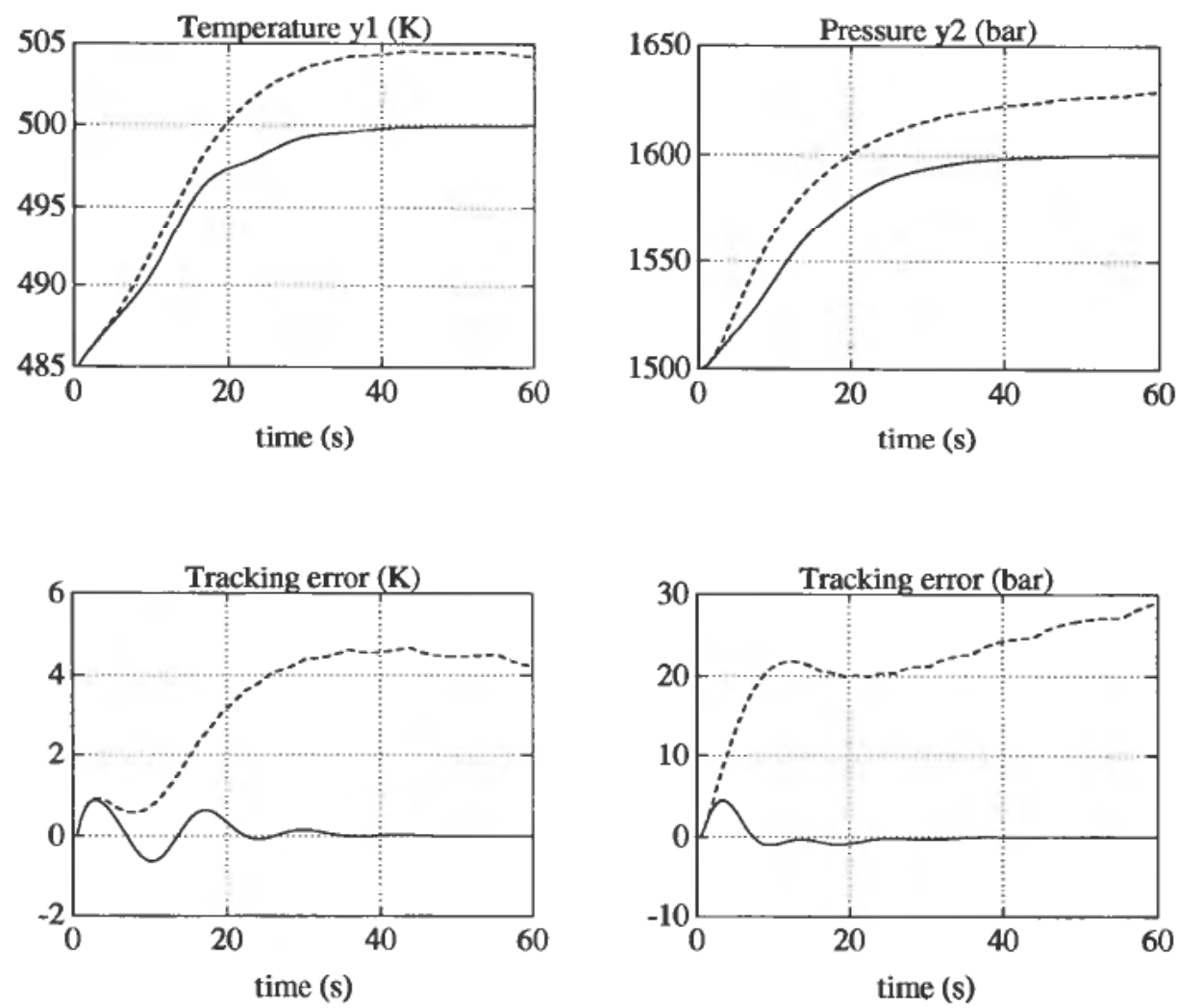

Figure 2. Time responses and tracking errors for the nominal controller (dashed) and the sliding controller (solid) given a commanded input vector: $r=[500(K), 1600(\text { bar })]^{T}$ for temperature and pressure, respectively.
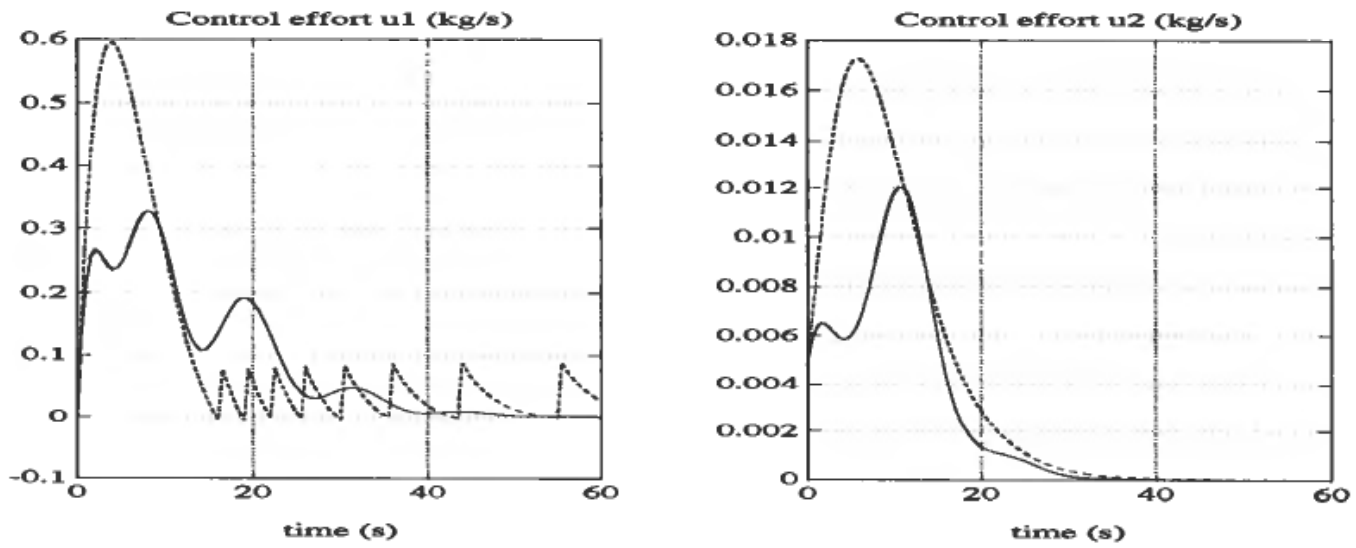

Figure 3. Control inputs for the nominal controller (dashed) and the sliding controller (solid). 
the sliding mode controller to be the superior one. It should be noted that the steady state error of the nominal controller could be eliminated by including integral action.

\section{Conclusions}

Sliding control of MIMO nonlinear minimum phase systems has been discussed. Model uncertainties are compensated for by adding a discontinuous term to the controller. Control of a polymerization reactor is used to illustrate the control scheme. Simulation results show that the sliding controller improves the performance significantly when model uncertainties are present.

\section{ACKNOWLEDGMENTS}

We thank Dr. Peter Singstad at the SINTEF Automatic Control for valuable discussions of the simulation results. This work was partially sponsored by the Royal Norwegian Council for Scientific and Industrial Research.

\section{References}

BYRNES, C. and IsIDORI, A. (1984). A frequency domain philosophy for nonlinear systems with application to stabilization and adaptive control, Proc. IEEE Conf. on Decision and Control, Las Vegas, Nevada, pp. 1569-1573.

SASTRY, S. S. and IsIDORI, A. (1991). Adaptive control of linearizable systems. IEEE Transactions on Automatic Control, 34, 1123-1131.

SinGSTAD, P. (1992). Modelling and control of an industrial autoclave LDPE polymerization reactor. Dring Thesis, The Norwegian Institute of Technology, Division of Engineering Cybernetics, N-7034 Trondheim, Norway.

SLOTINE, J. J. E. (1983). Tracking control of nonlinear systems using sliding surfaces. Ph.D Thesis, MIT Dept. of Aero. and Astrodynamics, Cambridge.

SLOTiNE, J. J. E. and LI, W. (1987). Adaptive manipulator control. A case study. IEEE Int. Conference on Robotics and Automation, pp. 1392-1401.

Slotine, J. J. E. and Li, W. (1991). Applied Nonlinear Control, Prentice-Hall.

YOERGER, D. R. and SLOTINE, J. E. (1985). Robust trajectory control of underwater vehicles. IEEE Journal of Oceanic Engineering, 10, 462-470.

UTKIN, I. U. (1977). Variable structure systems with sliding modes. IEEE Transactions on Automatic Control, 22, 222. 